\title{
Role of indoleamine 2,3-dioxygenase in ischemia- reperfusion injury of renal tubular epithelial cells
}

\author{
THEODOROS ELEFTHERIADIS*, GEORGIOS PISSAS*, SPYRIDON GOLFINOPOULOS, \\ VASSILIOS LIAKOPOULOS and IOANNIS STEFANIDIS
}

Department of Nephrology, Faculty of Medicine, University of Thessaly, 41110 Larissa, Greece

Received December 11, 2020; Accepted March 18, 2021

DOI: $10.3892 / \mathrm{mmr} .2021 .12111$

\begin{abstract}
The present study evaluated indoleamine 2,3-dioxygenase 1 (IDO) kinetics and how it affects cell survival during the two distinct phases of ischemia-reperfusion (I-R) injury. Primary renal proximal tubular epithelial cells (RPTECs) were cultured under anoxia or reoxygenation with or without the IDO inhibitor 1-DL-methyltryptophan, the aryl-hydrocarbon receptor (AhR) inhibitor $\mathrm{CH} 223191$ or the ferroptosis inhibitor $\alpha$-tocopherol. Using cell imaging, colorimetric assays, PCR and western blotting, it was demonstrated that IDO was upregulated and induced apoptosis during anoxia. The related molecular pathway entails tryptophan degradation, general control non-derepressible-2 kinase (GCN2K) activation, increased level of phosphorylated eukaryotic translation initiation factor $2 \alpha$, activating transcription factor (ATF) 4, ATF3, C/EBP homologous protein, phosphorylated p53, p53, Bax, death receptor-5 and eventually activated cleaved caspase-3. Reoxygenation also upregulated IDO, which, in this case, induced ferroptosis. The related molecular pathway encompasses kynurenine production, AhR activation, cytochrome p450 enzymes increase, reactive oxygen species generation and eventually ferroptosis. In conclusion, in RPTECs, both anoxia and reoxygenation upregulated IDO, which in turn induced GCN2K-mediated apoptosis and AhR-mediated ferroptosis. Since both phases of I-R injury share IDO upregulation as a common point, its inhibition may prove a useful therapeutic strategy for preventing or attenuating I-R injury.
\end{abstract}

Correspondence to: Professor Theodoros Eleftheriadis, Department of Nephrology, Faculty of Medicine, University of Thessaly, Biopolis, Mezourlo Hill, 41110 Larissa, Greece

E-mail: teleftheriadis@yahoo.com

*Contributed equally

Key words: ischemia-reperfusion, indoleamine 2,3-dioxygenase, apoptosis, ferroptosis, general control non-derepressible-2 kinase, aryl-hydrocarbon receptor

\section{Introduction}

Ischemia-reperfusion (I-R) injury plays a significant role in several human diseases, such as acute myocardial infarction, stroke and multiorgan failure (1). Not surprisingly, I-R injury is the most frequent cause of acute kidney injury with renal tubular epithelial cells being extremely vulnerable due to their high metabolic demands (2). Thus, delineating the molecular mechanisms that govern I-R injury deems a significant research issue, as it may lead to novel therapeutic strategies.

Indoleamine 2,3-dioxygenase 1 (IDO) is a rate-limiting enzyme that degrades tryptophan through the kynurenine pathway. IDO initially engaged immunologists' attention due to its immunomodulatory properties (3). IDO may activate two main pathways. Tryptophan depletion by increasing the uncharged tryptophanyl-tRNA activates the general control non-derepressible-2 kinase (GCN2K) (4-6). In parallel, the produced kynurenine activates the aryl-hydrocarbon receptor $(A h R)(7,8)$. However, the role of IDO seems to extend beyond the immune system. Experimentally, in the mouse kidney, it has been shown that I-R injury increases IDO expression, whereas IDO inhibition ameliorates kidney injury and preserves renal function (9). Nevertheless, the exact molecular mechanisms are still unknown. Also, in cultures of renal tubular epithelial cells subjected to reoxygenation, cell death depends on the activation of AhR (10). Since kynurenine is a known endogenous activator of AhR (7), the aforementioned reoxygenation-induced AhR activation might result from the reoxygenation-induced IDO upregulation and the subsequent kynurenine overproduction.

The present study evaluated the kinetics of IDO expression and its effect on cell survival in primary renal proximal tubular epithelial cells (RPTECs) subjected to I-R injury. I-R injury consists of two consecutive but pathophysiologically distinct phases. During ischemia, cell death ensues due to cell energy collapse. However, the setting alters during reoxygenation, as cell death results from overproduction of reactive oxygen species (ROS) (1). Notably, confirming the pathophysiological difference between the two phases of I-R injury, previous studies showed that during ischemia, RPTECs death ensues through apoptosis $(11,12)$. In contrast to this, reperfusion induces lipid peroxidation and ferroptotic cell death (12-14). 
To evaluate the effect of the two different phases of I-R injury on IDO kinetics and how the latter may affect RPTECs survival, the current study developed a proper cell culture system. RPTECs were cultured under anoxia to simulate ischemia. To imitate reperfusion, RPTECs were initially cultured under anoxia, then washed, fresh culture medium was added and cells were cultured under normoxic conditions. Whenever needed, the IDO inhibitor 1-DL-methyl-tryptophane (1-MT) (15), the AhR inhibitor CH223191 (16) or the ferroptosis inhibitor $\alpha$-tocopherol were used (17). The IDO-triggered molecular pathways that may induce cell apoptosis during anoxia or cell ferroptosis due to reoxygenation were evaluated.

\section{Materials and methods}

Cell culture and imaging. Primary C57BL/6 mouse RPTECs (cat. no. C57-6015; Cell Biologics, Inc.) were cultured in Complete Epithelial Cell Medium/w kit, supplemented with epithelial cell growth supplement (epithelial growth factor, insulin, transferrin, L-glutamine, selenium, fetal bovine serum, and antibiotics) (cat. no. M6621; Cell Biologics, Inc.). The aforementioned primary cells were differentiated, well-characterized passage one RPTECs. Cells were expanded in $75-\mathrm{cm}^{2}$ flasks, and passage three cells were used for the experiments.

Cells were seeded at a density of 10,000 cells per well in 96-well plates or at a density of 300,000 cells per well in 6-well plates and incubated for $16 \mathrm{~h}$ before the onset of anoxic conditions. To reduce oxygen levels to $<1 \%$, the GasPak ${ }^{\mathrm{TM}} \mathrm{EZ}$ Anaerobe Container System with Indicator (cat. no. 26001; BD Biosciences) was used. Cells within the anaerobic container were cultured at $37^{\circ} \mathrm{C}$. These anoxic conditions simulated ischemia (12).

An inverted microscope (Axiovert 40C; Carl Zeiss AG) and a digital camera (3MP USB2.0 Microscope Digital Camera; AmScope) with the related software (AmScope v. x64, 3.7.3036; AmScope) were used for cell imaging.

A reciprocal approach based on the cell imaging hard end-point of anoxia- or reoxygenation-induced cell death was used for selecting the appropriate time points. Cell imaging detected that the time required for cell death of untreated cells due to anoxia was $48 \mathrm{~h}$. Onset of reoxygenation experiments was at half of that time, which is after $24 \mathrm{~h}$. Notably, cell imaging did not detect a difference in confluency between the control cells and the cells subjected to $24 \mathrm{~h}$ of anoxia.

In reoxygenation experiments, cells were washed with Dulbecco's phosphate buffer saline (PBS) (Sigma-Aldrich; Merck KGaA), supplemented with fresh culture medium, and placed at $37^{\circ} \mathrm{C}$ in a humidified atmosphere containing $5 \% \mathrm{CO}_{2}$. These conditions imitate reperfusion (12). Cell imaging detected that the time required for the death of untreated cells due to reoxygenation was only $4 \mathrm{~h}$.

As live cells are required to conduct reliable experiments, the various parameters were evaluated at half of the time needed for severe deterioration of untreated cells under anoxia or reoxygenation. Thus, anoxia experiments were performed after $24 \mathrm{~h}$ of anoxia and reoxygenation experiments after $2 \mathrm{~h}$ of reoxygenation.

Whenever needed, cells were treated with $100 \mu \mathrm{M}$ IDO inhibitor 1-MT (Sigma-Aldrich; Merck KGaA), $3 \mu \mathrm{M}$ AhR inhibitor CH223191 (Sigma-Aldrich; Merck KGaA) or $100 \mu \mathrm{M}$ ferroptosis inhibitor $\alpha$-tocopherol (Sigma-Aldrich; Merck KGaA). In the anoxia experiments, such treatments started at the onset of the anoxic conditions. In the reoxygenation experiments, such treatments started at the beginning of the reoxygenation in previously untreated cells that were subjected to $24 \mathrm{~h}$ of anoxia.

IDO mRNA level. Cells were cultured in 6-well plates $(300,000$ cells per well) and were subjected or not to anoxia or reoxygenation. Total cellular RNA was isolated from RPTECs using the TRIzol ${ }^{\circledR}$ reagent (cat. no. 15596026; Invitrogen; Thermo Fisher Scientific, Inc.) according to the manufacturer's instructions. RNA concentration was measured on an EnSpire ${ }^{\circledR}$ Multimode Plate Reader (PerkinElmer, Inc.), and $5 \mu \mathrm{g}$ was used for first-strand cDNA synthesis using the PrimeScript ${ }^{\mathrm{TM}}$ II Reverse Transcriptase (cat. no. 2690A; Takara Bio, Inc.). RT was performed under the following conditions: $25^{\circ} \mathrm{C}$ for $5 \mathrm{~min}$, $42^{\circ} \mathrm{C}$ for $60 \mathrm{~min}$ and $70^{\circ} \mathrm{C}$ for $15 \mathrm{~min}$. The PCR platform used was an Eppendorf Reaplex 4 MasterCycler (Eppendorf). The resultant cDNA samples were subjected to 30 cycles of PCR amplification in the presence of specific sense and antisense primers for mouse IDO and glyceraldehyde 3-phosphate dehydrogenase (GAPDH) as an internal control. The following thermocycling conditions were used: Initial denaturation step at $94^{\circ} \mathrm{C}$ for $2 \mathrm{~min}$; followed by 30 cycles of annealing at $60^{\circ} \mathrm{C}$ for $50 \mathrm{sec}$, elongation at $72^{\circ} \mathrm{C}$ for $1 \mathrm{~min}$ and denaturation at $94^{\circ} \mathrm{C}$ for $30 \mathrm{sec}$. The primer sequences used were as follows: IDO sense, 5'-AGGATCCTTGAAGACCACCA-3' and antisense, 5'-CCAATAGAGAGACGAGGAAG-3' (398 bp); and GAPDH sense, 5'-GCCAAGGTCATCCATGACAACTTTGG-3' and antisense, 5'-GCCTGCTTCACCACCTTCTTGATGTC-3' (348 bp). Primers were obtained from Eurofins Scientific. The amplified PCR products were electrophoresed in a $1.0 \%$ agarose gel (cat. no. A9539; Sigma-Aldrich; Merck KGaA) and stained with ethidium bromide (cat. no. 1116150001; Sigma-Aldrich; Merck KGaA). Digital images were obtained with a Kodak digital device (Kodak). Densitometric analysis was performed using Image J software version 1.53f (National Institutes of Health). These experiments were repeated three times.

Cell death, ROS production, tryptophan catabolism and kynurenine production. Besides cell imaging, cell death was also assessed in cells cultured in 96-well plates $(10,000$ cells per well) by LDH release using the Cytotox Non-Radioactive Cytotoxic Assay kit (cat. no. G1780; Promega Corporation) according to the manufacturer's protocol. The LDH release assay was performed to detect both necrotic and apoptotic death (18). Cell death was calculated using the following equation: Cell death $(\%)=(\mathrm{LDH}$ in the supernatant: total LDH) x100. These experiments were repeated six times.

ROS generation was evaluated in cells cultured in 96-well plates (10,000 cells per well). Once the incubation period was over, $5 \mu \mathrm{M}$ fluorogenic probe CellROX ${ }^{\circledR}$ Deep Red Reagent (cat. no. C10422; Invitrogen; Thermo Fisher Scientific, Inc.) was added for $30 \mathrm{~min}$ at $37^{\circ} \mathrm{C}$. The cells were then washed with PBS, and fluorescence signal intensity was measured on an EnSpire ${ }^{\circledR}$ Multimode Plate Reader (PerkinElmer, Inc.). These experiments were repeated six times.

Tryptophan catabolism and kynurenine production were assessed by their concentration in the cell culture supernatant. Cells were cultured in 6-well plates $(300,000$ cells per well). Once the incubation period was over, tryptophan 
A
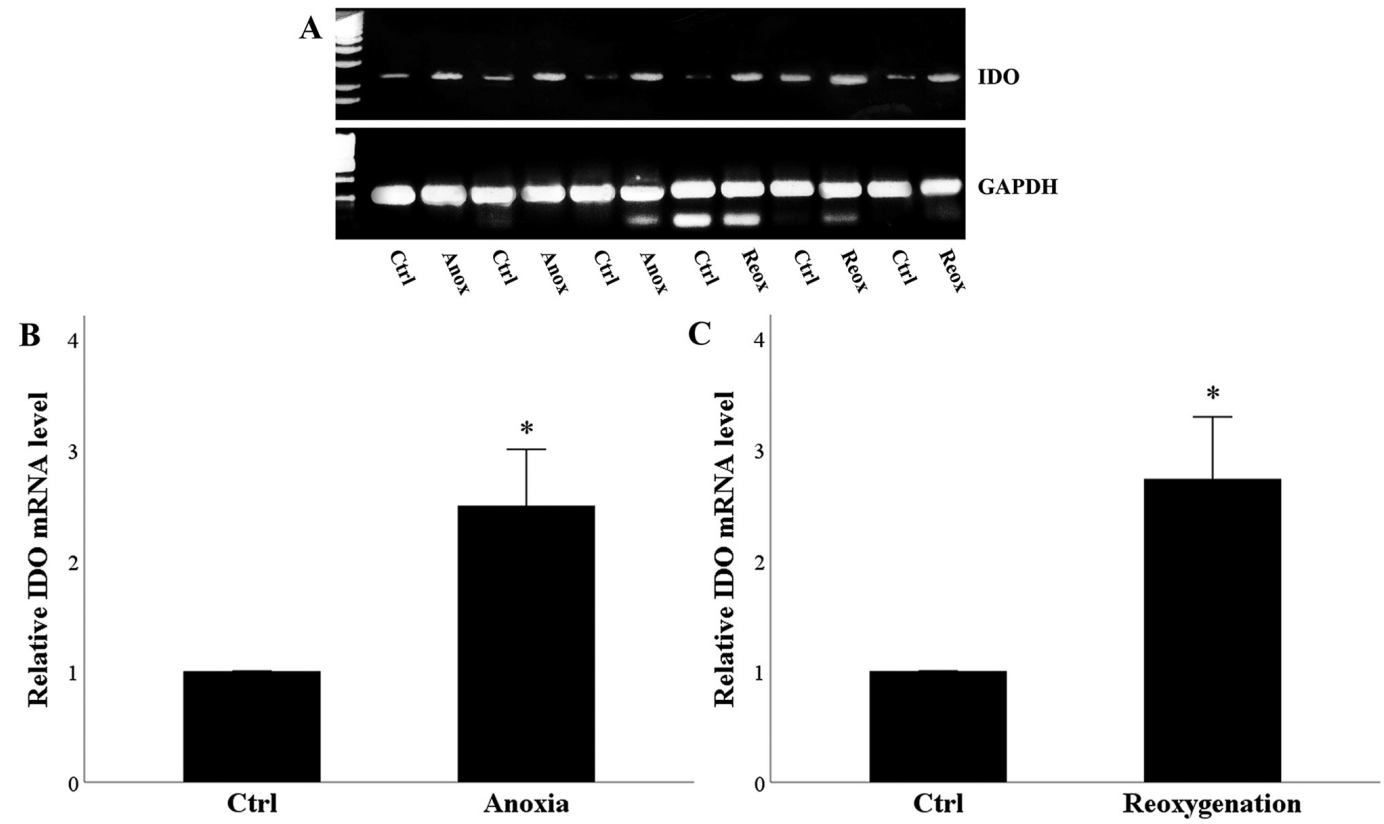

Figure 1. Effect of anoxia or reoxygenation on IDO mRNA level. (A and B) Reverse transcription semi-quantitative PCR showed that anoxia increased IDO mRNA significantly. (C) Relative IDO mRNA levels in cells subjected to reoxygenation. " $\mathrm{P}<0.05$ vs. Control cells. IDO, indoleamine 2,3-dioxygenase; GAPDH, glyceraldehyde 3-phosphate dehydrogenase; Ctrl, control.

and kynurenine concentrations were measured using the kynurenine/tryptophan ratio ELISA pack (cat. no. ISE-2227; ImmuSmol). Limits of detections of the kit for kynurenine and tryptophan are below $47.5 \mathrm{ng} / \mathrm{ml}$ and $1.2 \mu \mathrm{g} / \mathrm{ml}$, respectively. These experiments were repeated six times.

Proteins of interest. Once anoxia or reoxygenation period was over, RPTECs cultured in 6-well plates $(300,000$ cells per well) were lysed with the T-PER tissue protein extraction reagent (Thermo Fisher Scientific, Inc.) supplemented with protease (Sigma-Aldrich; Merck KGaA) and phosphatase inhibitors (Roche Diagnostics). Bradford assay (Sigma-Aldrich; Merck $\mathrm{KGaA}$ ) was used for protein quantification, and $10 \mu \mathrm{g}$ from each protein extract was loaded per lane and electrophoresed on an SDS-PAGE gel (4-12\% Bis-Tris gels; Thermo Fisher Scientific, Inc.) and transferred onto a polyvinylidene fluoride (PVDF) membrane (Thermo Fisher Scientific, Inc.). The LumiSensor Plus Chemiluminescent HRP Substrate kit (GenScript) was used for the enhanced chemiluminescent detection of the western blot bands. The Restore Western Blot Stripping Buffer (Thermo Fisher Scientific, Inc.) was used for re-probing the PVDF membranes. Densitometric analysis was performed with the ImageJ software. These experiments were repeated four times.

Blots were incubated at $4^{\circ} \mathrm{C}$ for $16 \mathrm{~h}$ with each primary antibody and for $30 \mathrm{~min}$ at room temperature with the appropriate secondary antibody. Primary antibodies were specific the following proteins: IDO (1:200; cat. no. sc-25809), GCN2K (1:100; cat. no. sc-374609) (both from Santa Cruz Biotechnology, Inc.), phosphorylated at Thr899 GCN2K (p-GCN2K; 1:1,000; cat. no. ab75836; Abcam), eukaryotic translation initiation factor-2 $\alpha$ (eIF $2 \alpha$; 1:100; cat. no. sc-133132; Santa Cruz Biotechnology, Inc.), p at Ser51 eIF2 $\alpha$ (p-eIF2 $\alpha$; 1:1,000; cat. no. 9721; Cell Signaling Technology, Inc.), activating transcription factor 4 (ATF4; 1:500; cat. no. CSB-PA002272KA01HU), ATF3
(1:500; cat. no. CSB-PA020022) (both Cusabio Technology LLC), C/EBP homologous protein (CHOP; 1:1,000; cat. no. 5554), p53 (1:1,000; cat. no. 2524), p at Ser15 p53 (p-p53; 1:1,000; cat. no. 9284), Bax (1:1,000; cat. no. 5023) (all Cell Signaling Technology, Inc.), death receptor 5 (DR5; 1:500; cat. no. CSB-PA018500; Cusabio Technology LLC), activated cleaved caspase-3 (CC3; 1:1,000; cat. no. ab13847; Abcam), AhR (1:200; cat. no. sc-133088), cytochrome P450 family 1 subfamily A polypeptide 1 (CYP1A1; 1:500; cat. no. sc-25304) (both Santa Cruz Biotechnology, Inc.) and $\beta$-actin (1:2,500; cat. no. 4967; Cell Signaling Technology, Inc.). Anti-mouse (1:1,000; cat. no. 7076$)$ or anti-rabbit $(1: 1,000$, cat. no. 7074) (both Cell Signaling Technology, Inc.) IgG HRP-conjugated secondary antibodies were used.

Statistical analysis. Statistical analysis was performed with SPSS software version 20 (IBM Corp.). The one-sample Kolmogorov-Smirnov test verified that all variables were normally distributed except the cell imaging results. An unpaired Student's t-test or one-way ANOVA with Bonferroni's post hoc test were used for comparison of means. For analyzing the cell imaging results, the Mann-Whitney U test or the Kruskal-Wallis H test with Dunn's post hoc test were used. Results are expressed as the mean \pm SEM, and $\mathrm{P}<0.05$ was considered to indicate a statistically significant difference. Western blotting results were normalized against $\beta$-actin and PCR results were normalized against GAPDH.

\section{Results}

Anoxia or reoxygenation increases IDO $\mathrm{mRNA}$ expression. RPTECs remained under normoxic conditions for $24 \mathrm{~h}$ or subjected to $24 \mathrm{~h}$ of anoxia. Compared with the control cells, anoxia increased IDO mRNA level significantly (Fig. 1A and B). Control RPTECs remained under 


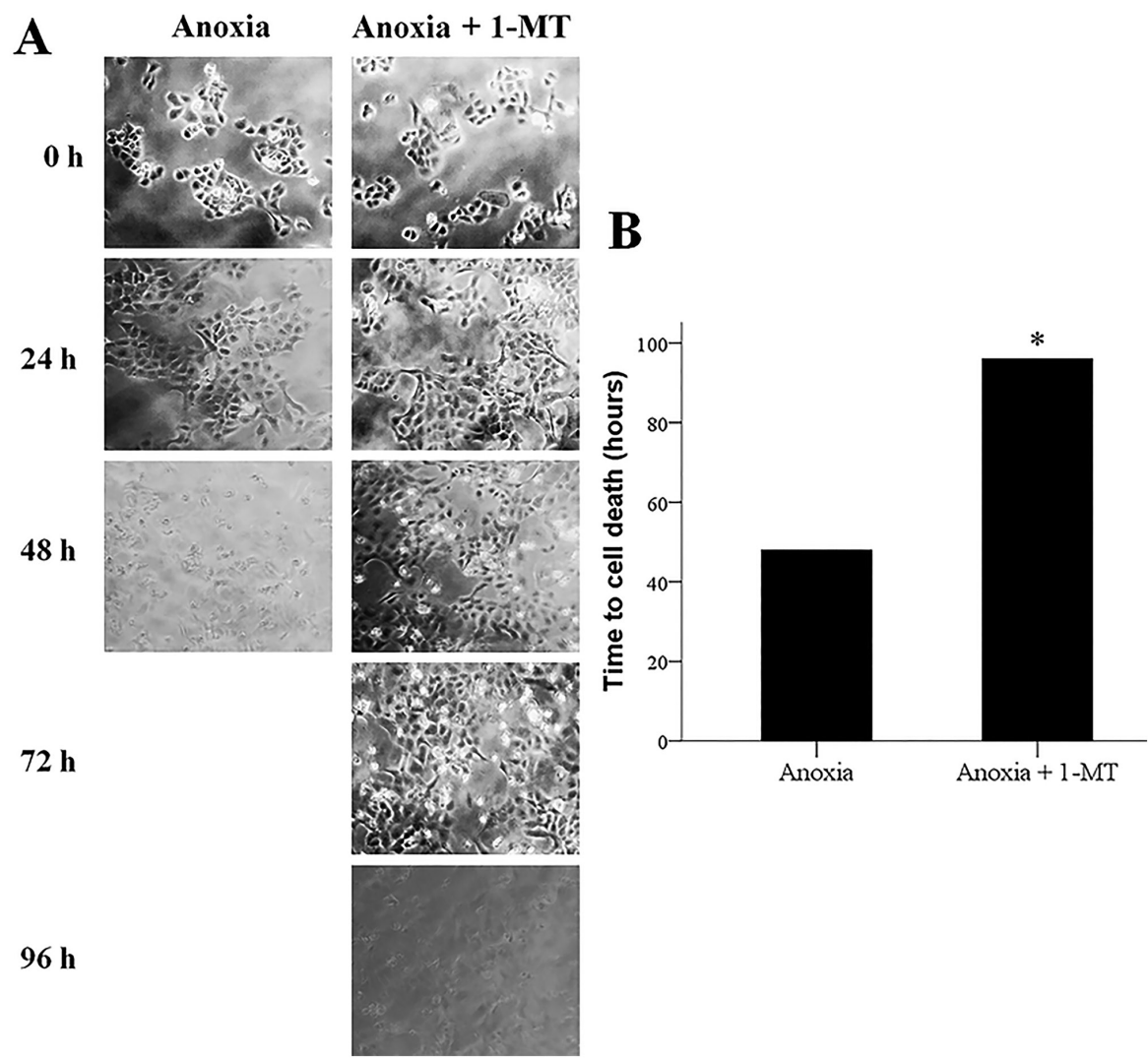

Figure 2. Anoxia-induced cell death and the effect of IDO inhibition. (A) Representative cell imaging (magnification, x100) showed that RPTECs are vulnerable to anoxia-induced cell death, while the IDO inhibitor 1-MT rescues RPTECs. (B) Cumulative results of six repeated experiments. "P<0.05 vs. anoxia. 1-MT, 1-DL-methyltryptophan; IDO, indoleamine 2,3-dioxygenase 1; RPTECs, renal proximal tubular epithelial cells.

normoxic conditions for $24 \mathrm{~h}$, washed and remained for another $2 \mathrm{~h}$ period under normoxia before mRNA extraction. Treated RPTECs were subjected to $24 \mathrm{~h}$ of anoxia, and then washed and cultured for another 2 -h period under normoxic conditions. Compared with the control cells, reoxygenation enhanced IDO mRNA level significantly (Fig. 1A and C). Thus, both anoxia and reoxygenation increased the mRNA expression of IDO.

Inhibition of IDO prevents anoxia-induced apoptosis. Cell imaging revealed that anoxia induced cell death, whereas the IDO inhibitor 1-MT prevented anoxia-induced cell death (Fig. 2A and B). Western blotting showed that anoxia increased the level of activated $\mathrm{CC} 3$, on which all the apoptotic pathways converge (19). The IDO inhibitor 1-MT prevented anoxia-induced CC3 upregulation (Fig. 3A and B).

Possibly due to lack of oxygen, anoxia did not upregulate ROS generation in the presence or not of 1-MT (Fig. 3C). The LDH release assay confirmed the results obtained by cell imaging, demonstrating that anoxia induces cell death, whereas the IDO inhibitor 1-MT decreases anoxia-induced cell death. Notably, the ferroptosis inhibitor $\alpha$-tocopherol did not affect anoxia-induced cell death, ensuring that ferroptosis does not occur under anoxia (Fig. 3D). The latter is in accordance with the aforementioned stable ROS levels since ROS overproduction is required for ferroptosis (17). Hence, during anoxia, cell death ensues through apoptosis, whereas ferroptosis does not take place. Inhibition of IDO could prevent anoxia-induced apoptotic cell death.
Anoxia upregulates IDO, depletes tryptophan and activates $G C N 2 K$. Anoxia induced IDO overexpression, a result that was not affected by the IDO inhibitor 1-MT (Fig. 4A and D). Tryptophan catabolism was assessed by its concentration in the cell culture supernatant. The results confirmed that anoxia-induced IDO-overexpression decreased tryptophan concentration significantly, whereas 1-MT prevents tryptophan depletion (Fig. 4E).

Tryptophan depletion activated GCN2K as it was assessed by the level of its activated phosphorylated form. As expected, the absence of tryptophan depletion in cells treated with 1-MT prevented anoxia-induced GCN2K activation (Fig. 4B and F). Anoxia-induced GCN2K activation led to increased phosphorylated eIF2 $\alpha$, a substrate of the GCN2K (6). In 1-MT-treated cells, the absence of GCN2K activation prevented anoxia-induced eIF $2 \alpha$ phosphorylation (Fig. 4C and G). Therefore, anoxia increased the protein expression of IDO. IDO could deplete tryptophan and activate GCN2K. 1-MT inhibited IDO activity and prevented $\mathrm{GCN} 2 \mathrm{~K}$ activation.

IDO-induced GCN2 activation triggers apoptotic pathways. Under anoxia, the p-eIF2 $\alpha$ translational target ATF4 was upregulated, whereas 1-MT prevented anoxia-induced ATF4 upregulation (Fig. 5A and F). In cells subjected to anoxia, the ATF4 transcriptional target CHOP increased, while the IDO inhibitor 1-MT blocked anoxia-induced CHOP upregulation (Fig. 5B and G).

Under anoxia, ATF3, a transcriptional target of ATF4, expression increased, whereas 1-MT prevented 

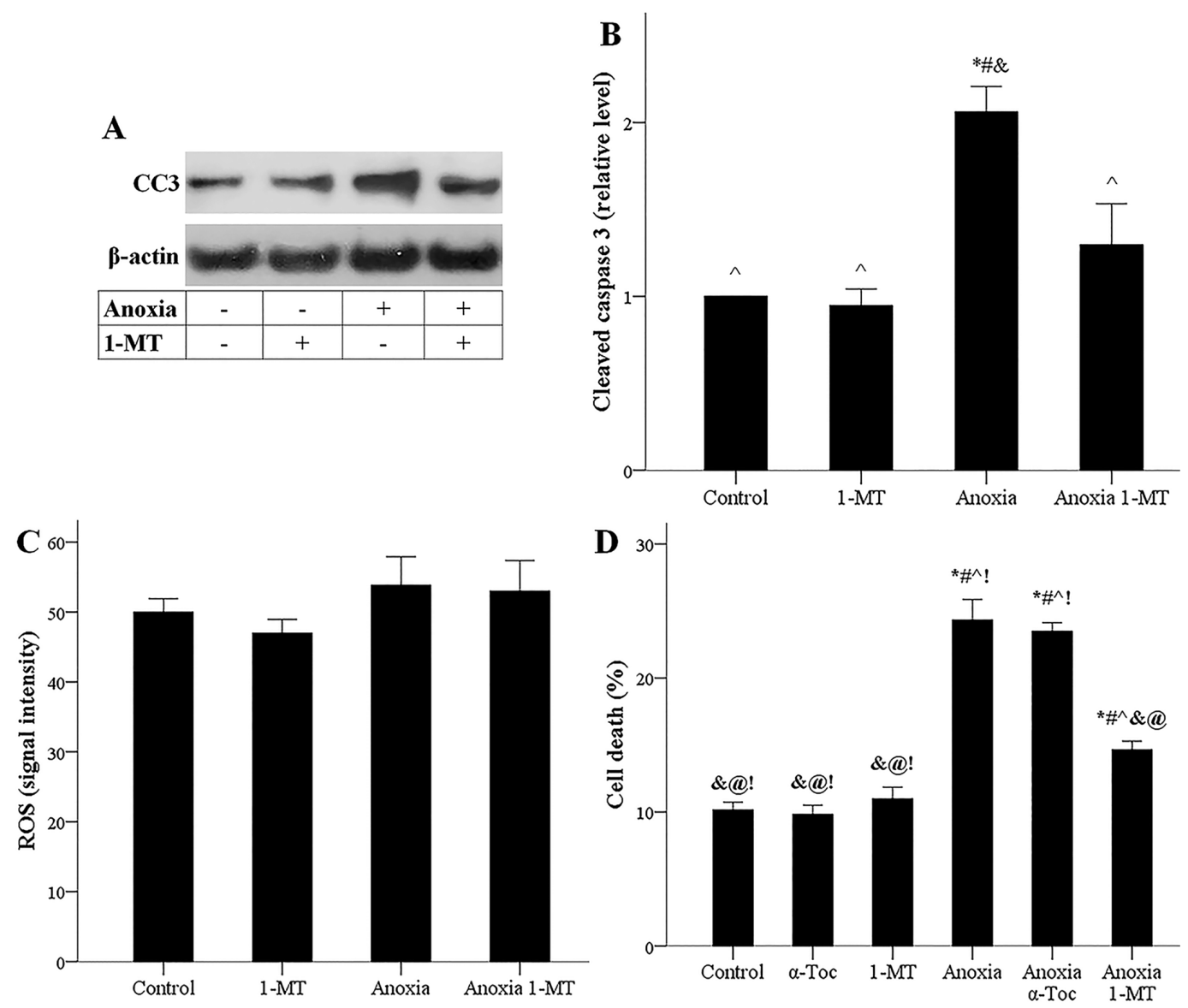

Figure 3. Effect of anoxia in the presence or absence of the IDO inhibitor 1-MT on CC3, ROS production and ferroptotic cell death. (A) Representative western blotting of $\mathrm{CC} 3$ levels and (B) semi-quantification. ${ }^{*} \mathrm{P}<0.05$ vs. control; ${ }^{\#} \mathrm{P}<0.05$ vs. control with $1-\mathrm{MT}$; ${ }^{\wedge} \mathrm{P}<0.05$ vs. anoxia; ${ }^{\circledR} \mathrm{P}<0.05$ vs. anoxia with 1-MT. (C) ROS levels with or without 1-MT treatment and anoxia. (D) LDH release assay indicating cell death. "P<0.05 vs. control; " $\mathrm{P}<0.05$ vs. control with $\alpha$-Toc; ${ }^{\wedge} \mathrm{P}<0.05$ vs. control with 1 -MT; ${ }^{\&} \mathrm{P}<0.05$ vs. anoxia; ${ }^{\circledR} \mathrm{P}<0.05$ vs. anoxia with $\alpha-$ Toc; $\mathrm{P}<0.05$ vs. anoxia with 1 -MT. 1 -MT, 1 -DL-methyltryptophan; $\alpha$-Toc, $\alpha$-tocopherol; CC3, cleaved caspase-3; IDO, indoleamine 2,3-dioxygenase 1; ROS, reactive oxygen species.

anoxia-induced ATF3 upregulation (Fig. 5C and H). Anoxia induced phosphorylation of $\mathrm{p} 53$, an effect that was reduced significantly by 1-MT (Fig. 5D and I). In cells subjected to anoxia, the increase of ATF3 and p-p53 was accompanied by $\mathrm{p} 53$ upregulation, while 1-MT prevented anoxia-induced p53 upregulation (Fig. 5E and J). The p-p53 to total p53 ratio remained stable under all conditions (Fig. 5K), indicating that phosphorylation of p53 at Ser15 controls total p53 level.

Anoxia-induced increase of CHOP and p53 was accompanied by elevated levels of their transcriptional target Bax. 1-MT inhibited anoxia-induced Bax upregulation (Fig. 6A and B). Also, anoxia-induced increase of CHOP and p53 was accompanied by enhanced expression of their transcriptional target DR5. 1-MT blocked anoxia-induced DR5 upregulation as well (Fig. 6C and D). Thus, anoxia-induced GCN2K activation could trigger the activation of apoptotic pathways, whereas inhibition of IDO prevented the activation of the aforementioned apoptotic pathways.

Inhibition of IDO prevents reoxygenation-induced ferroptosis. Cell imaging showed that reoxygenation induced cell death, while both 1-MT and the AhR inhibitor CH223191 prevented reoxygenation-induced cell death (Fig. 7A and B). Meanwhile, irrespective of the presence of 1-MT or CH223191, reoxygenation did not affect the level of activated $\mathrm{CC} 3$, indicating that under reoxygenation, apoptosis does not take place (Fig. 8A and B).

The LDH release assay confirmed the results obtained by cell imaging results, since it detected that reoxygenation induces cell death, while 1-MT prevented reoxygenation-induced cell death. CH223191 also prevented reoxygenation-induced cell death, indicating that, under reoxygenation, cell death is mediated by AhR. Notably, the ferroptosis inhibitor $\alpha$-tocopherol blocked reoxygenation-induced cell death, confirming the ferroptotic nature of cell death under reoxygenation (Fig. 8C). Therefore, reoxygenation induced ferroptotic cell death, whereas apoptosis did not take place, and the results indicated that ferroptosis was mediated by IDO and AhR.

Reoxygenation upregulates IDO, increases kynurenine and triggers AhR-induced ROS generation. Reoxygenation upregulated IDO expression, while neither 1-MT nor CH223191 affected IDO (Fig. 9A and B). In cells subjected to reoxygenation, IDO-overexpression was accompanied by increased kynurenine production assessed by its concentration in the cell culture supernatant. Inhibition of IDO mediated by 1-MT decreased reoxygenation-induced kynurenine-overproduction, while the AhR inhibitor CH223191 did not affect kynurenine levels (Fig. 9C).

The reoxygenation-induced kynurenine-overproduction was accompanied by a reduction in AhR levels. Reduction 
A

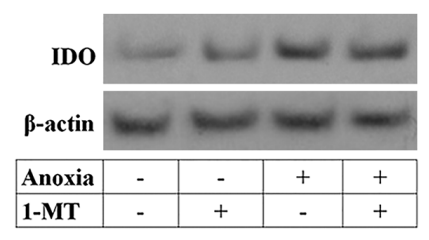

B

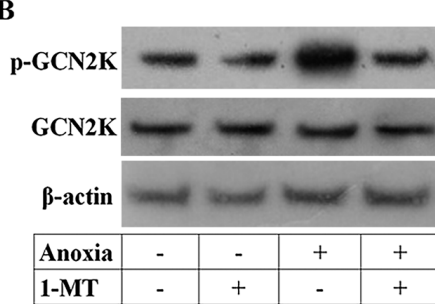

E
C

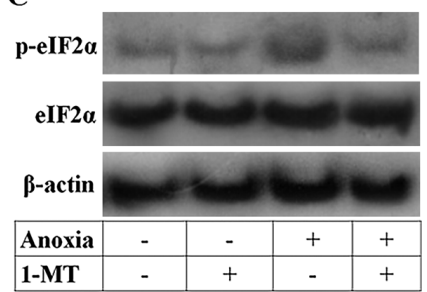

D

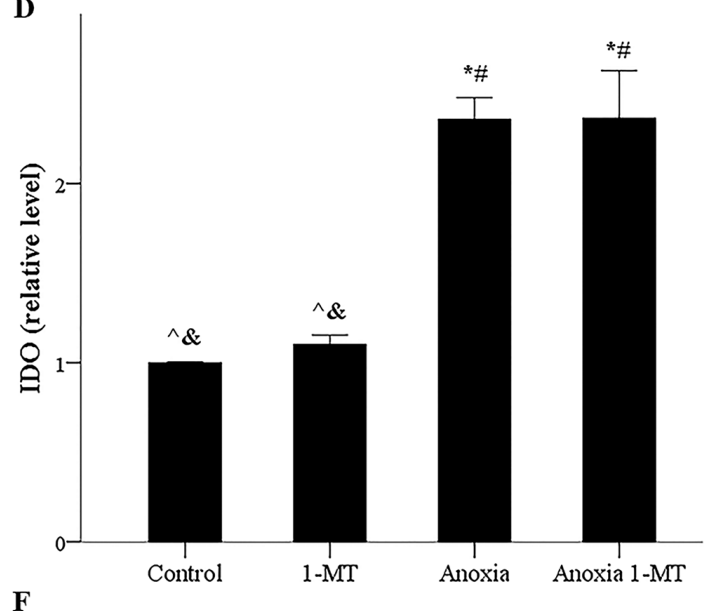

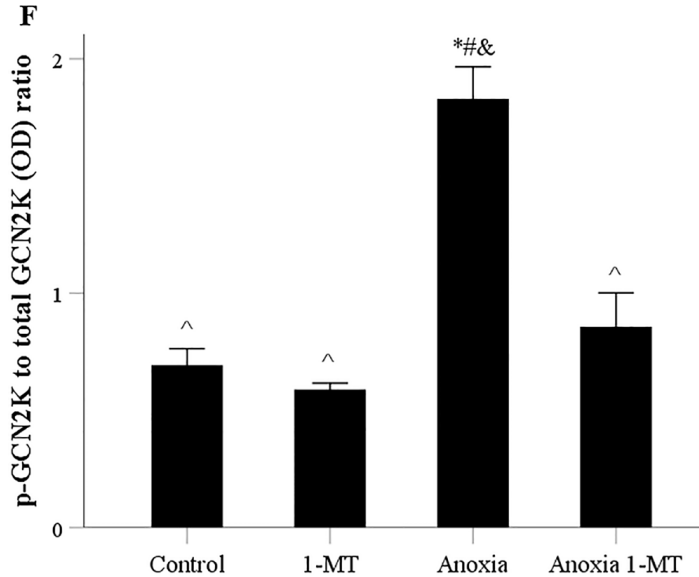

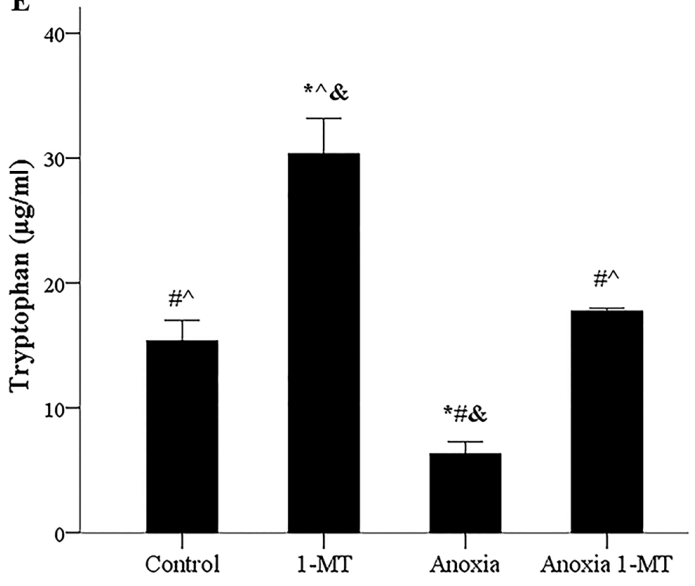

$\mathbf{G}$

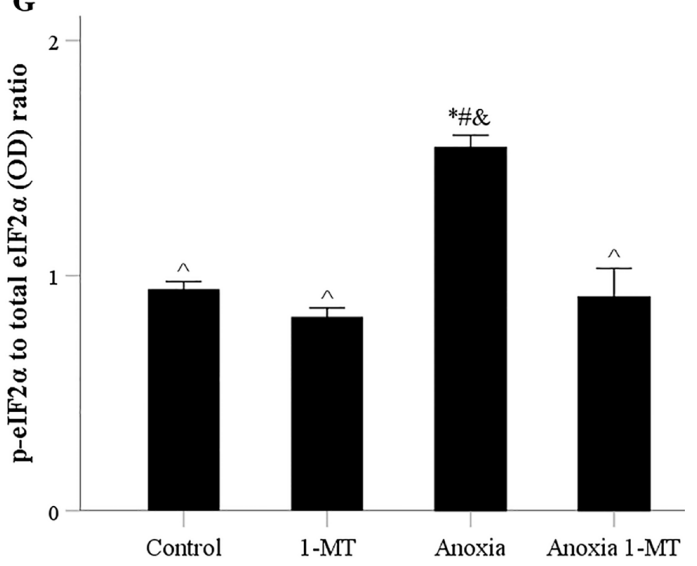

Figure 4. Effect of anoxia in the presence or absence of the IDO inhibitor 1-MT on IDO expression, tryptophan catabolism, p-GCN2K and p-eIF2 $\alpha$ levels. Representative western blotting of (A) IDO, (B) p- and total GCN2K and (C) p- and total eIF2 $\alpha$ levels. (D) Semi-quantification of IDO protein levels. (E) Tryptophan catabolism was assessed by its concentration in the cell culture supernatant. (F) p-GCN2K/GCN2K and (G) p-eIF2 $\alpha /$ eIF $2 \alpha$ ratios. ${ }^{*} \mathrm{P}<0.05$ vs. control; " $\mathrm{P}<0.05$ vs. control with $1-\mathrm{MT}$; ${ }^{\wedge} \mathrm{P}<0.05$ vs. anoxia; ${ }^{\circledR} \mathrm{P}<0.05$ vs. anoxia with 1-MT. 1-MT, 1-DL-methyltryptophan; IDO, indoleamine 2,3-dioxygenase 1; p-, phosphorylated; GCN2K, general control nonderepressible-2 kinase; eIF2 $\alpha$, eukaryotic translation initiation factor $2 \alpha$; OD, optical density.

in AhR levels reflects the activation status of the receptor as only the activated form of AhR is targeted by proteasomal degradation leading to decreased AhR cellular levels $(20,21)$. Under the reoxygenation conditions, both 1-MT, which by inhibiting IDO prevents kynurenine production $(7,8,15)$, and CH223192, which inhibits AhR directly (16), prevented AhR activation (Fig. 10A and B). In cells subjected to reoxygenation, activated AhR led to an enhanced expression of its transcriptional target, CYP1A1. 1-MT decreased reoxygenation-induced CYP1A1-overexpression possibly by reducing kynurenine-induced AhR activation. As expected, the AhR inhibitor CH223191 also decreased reoxygenation-induced CYP1A1-overexpression (Fig. 10C and D). In cells subjected to reoxygenation, CYP1A1 upregulation was accompanied by $\mathrm{RO}$-overproduction, a prerequisite for ferroptotic cell death (17). Both 1-MT and $\mathrm{CH} 223191$ blocked reoxygenation-induced ROS-overproduction (Fig. 10E).

\section{Discussion}

Triggered by experimental data that showed upregulation of IDO in a model of kidney I-R injury and a beneficial effect of IDO inhibition (9), the present study evaluated IDO kinetics and the subsequent molecular pathways that may affect cell survival under anoxia or reoxygenation in RPTECs.

In accordance with a previous study (12), the current results showed that anoxia induced apoptotic and not ferroptotic cell death as anoxia did not increase ROS generation, which is a prerequisite for ferroptosis (17). Anoxia increased IDO mRNA and protein expression. The subsequent tryptophan depletion led to autophosphorylation and activation of the GCN2K, likely due to increased uncharged tryptophanyl-tRNA (6). GCN2K phosphorylated its substrate eIF2 $\alpha$, which is known to alter the cellular translational program (6). In accordance with previous studies $(6,22)$, p-eIF2 $\alpha$ enhanced 

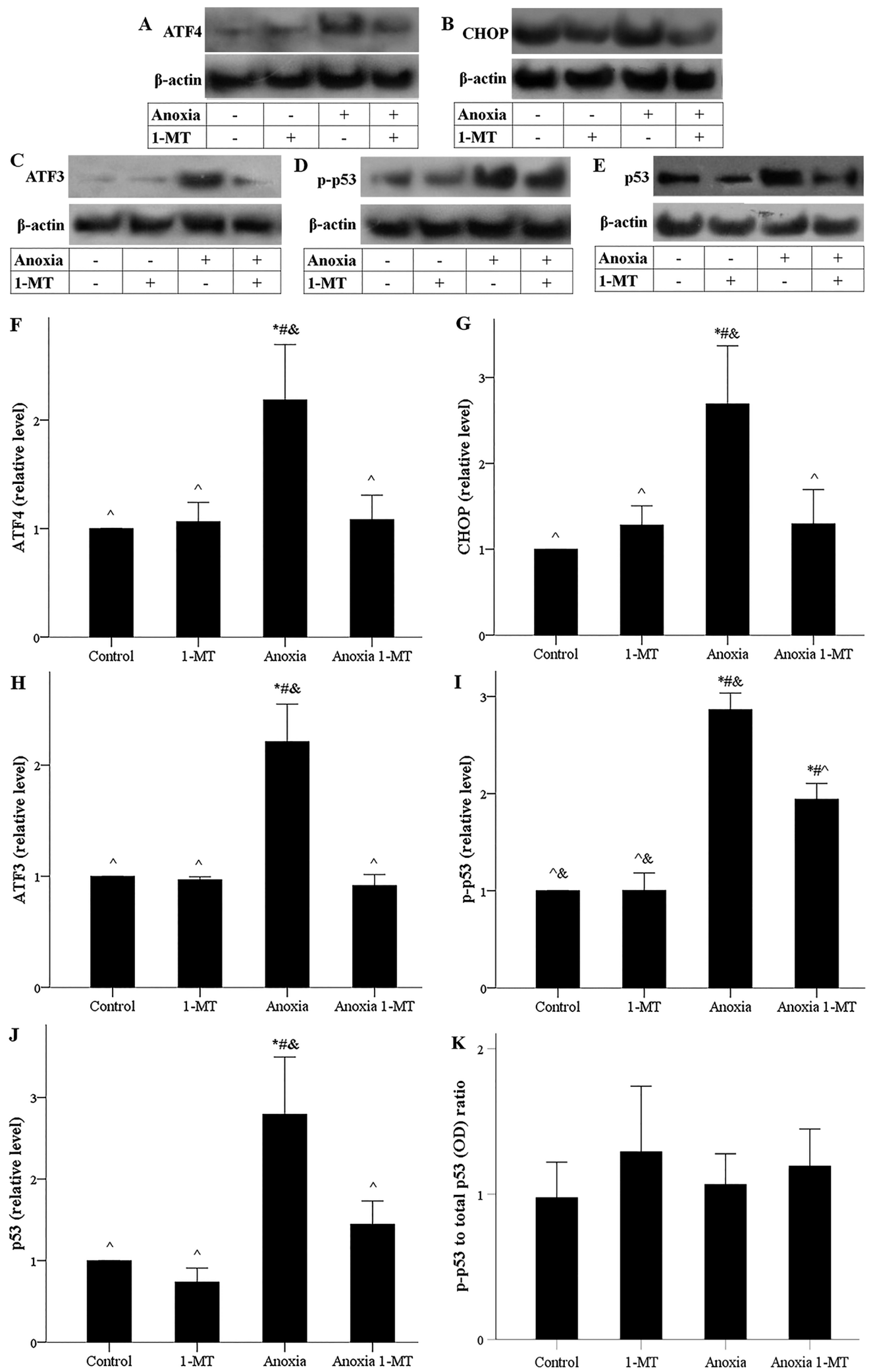

Figure 5. Effect of anoxia in the presence or absence of the IDO inhibitor 1-MT on ATF4, CHOP, ATF3, p-p53 and p53 levels. Representative western blots for the levels of (A) ATF4, (B) CHOP, (C) ATF3, (D) p-p53 and (E) p53. Semi-quantification of (F) ATF4, (G) CHOP, (H) ATF3, (I) p-p53 and (J) p53 protein levels. (K) p-p53/total p53 ratio. ${ }^{*} \mathrm{P}<0.05$ vs. control; " $\mathrm{P}<0.05$ vs. control with 1 -MT; ${ }^{\wedge} \mathrm{P}<0.05$ vs. anoxia; ${ }^{\circledR} \mathrm{P}<0.05$ vs. anoxia with 1-MT. 1-MT, 1-DL-methyltryptophan; IDO, indoleamine 2,3-dioxygenase 1; ATF4, activating transcription factor 4; CHOP; C/EBP homologous protein; ATF4, activating transcription factor 3; p-, phosphorylated; OD, optical density.

ATF4, which in turn upregulated the expression of its transcriptional target CHOP. CHOP is known to upregulate pro-apoptotic factors involved in both the intrinsic and extrinsic apoptotic pathways (22). Two pro-apoptotic factors were also evaluated in the present study. Anoxia-induced increased expression of CHOP, Bax and DR5. By increasing 

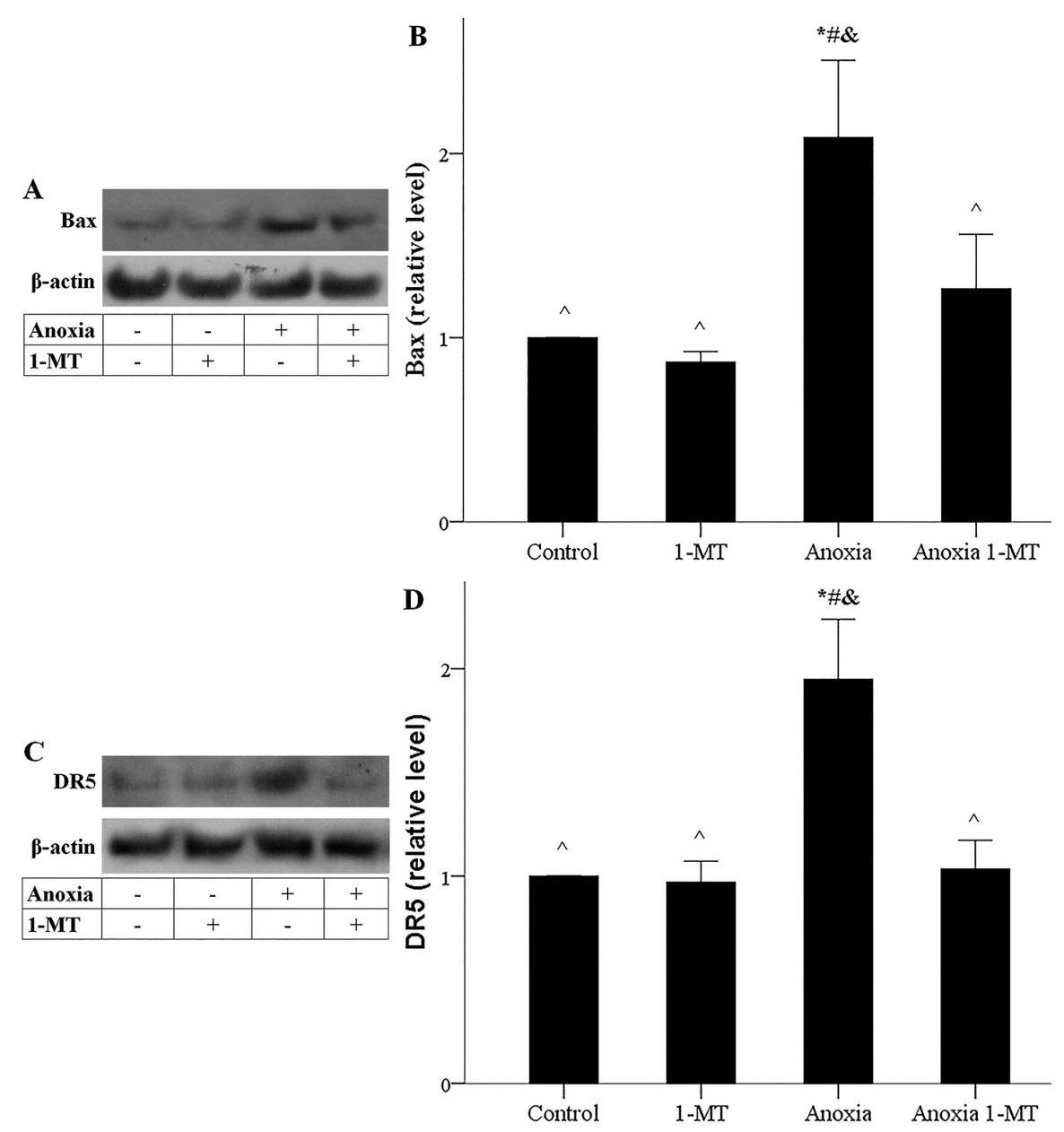

Figure 6. Effect of anoxia in the presence or absence of the IDO inhibitor 1-MT on Bax and DR5. (A) Bax protein levels and (B) semi-quantification. (C) DR5 protein levels and (D) semi-quantification. " $\mathrm{P}<0.05$ vs. control; ${ }^{*} \mathrm{P}<0.05$ vs. control with $1-\mathrm{MT}$; ${ }^{\wedge} \mathrm{P}<0.05$ vs. anoxia; ${ }^{\circledR} \mathrm{P}<0.05$ vs. anoxia with 1 -MT. 1-MT, 1-DL-methyltryptophan; IDO, indoleamine 2,3-dioxygenase 1; DR5, death receptor 5.

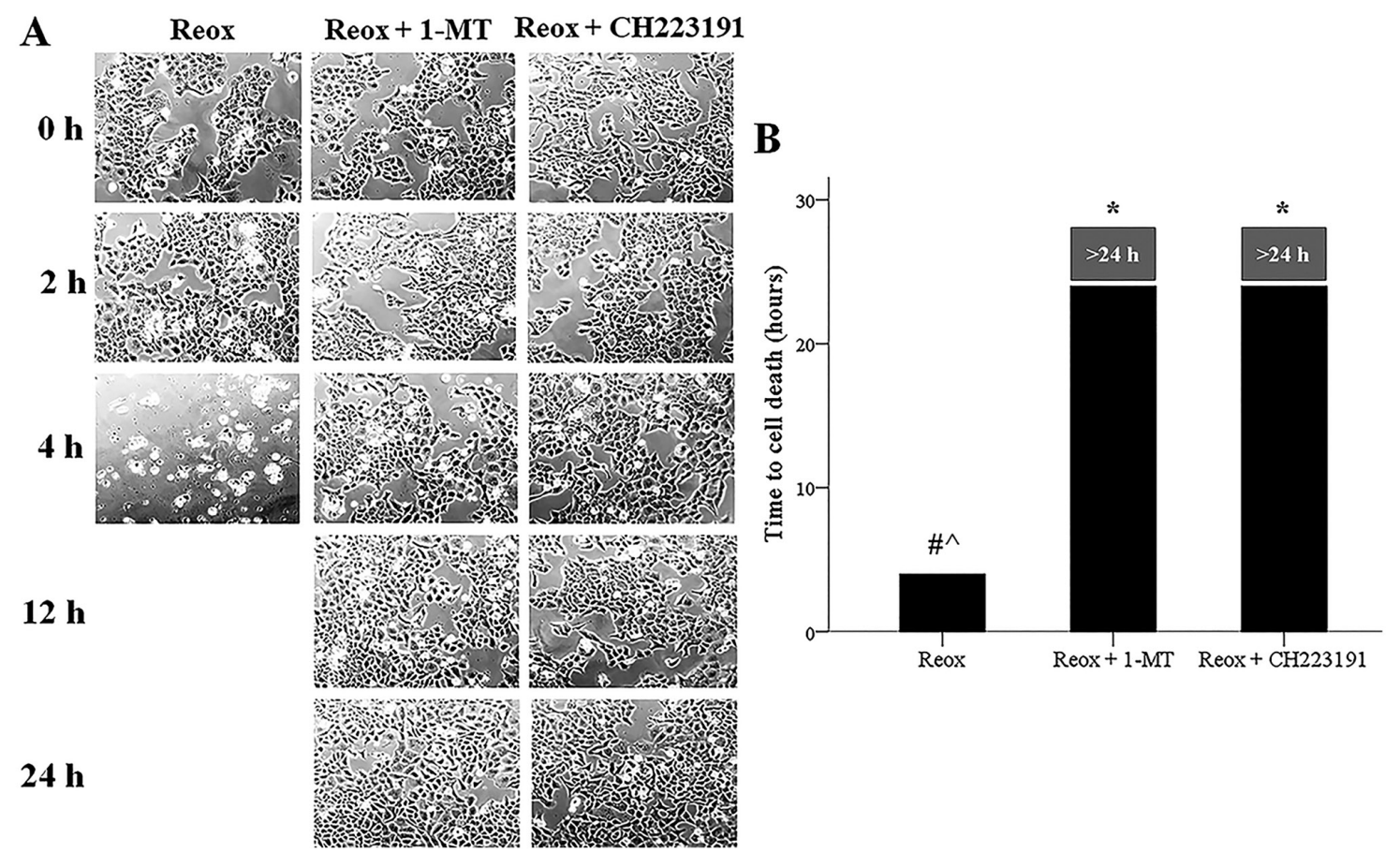

Figure 7. Reox-induced cell death and the effect of IDO or AhR inhibition. (A) Representative cell images (magnification, x100) and (B) quantification. "P<0.05 vs. Reox; ${ }^{*} \mathrm{P}<0.05$ vs. Reox with 1-MT; ${ }^{\wedge} \mathrm{P}<0.05$ vs. Reox with CH223191. 1-MT, 1-DL-methyltryptophan; AhR, aryl-hydrocarbon receptor; IDO, indoleamine 2,3-dioxygenase 1; Reox, reoxygenation. 

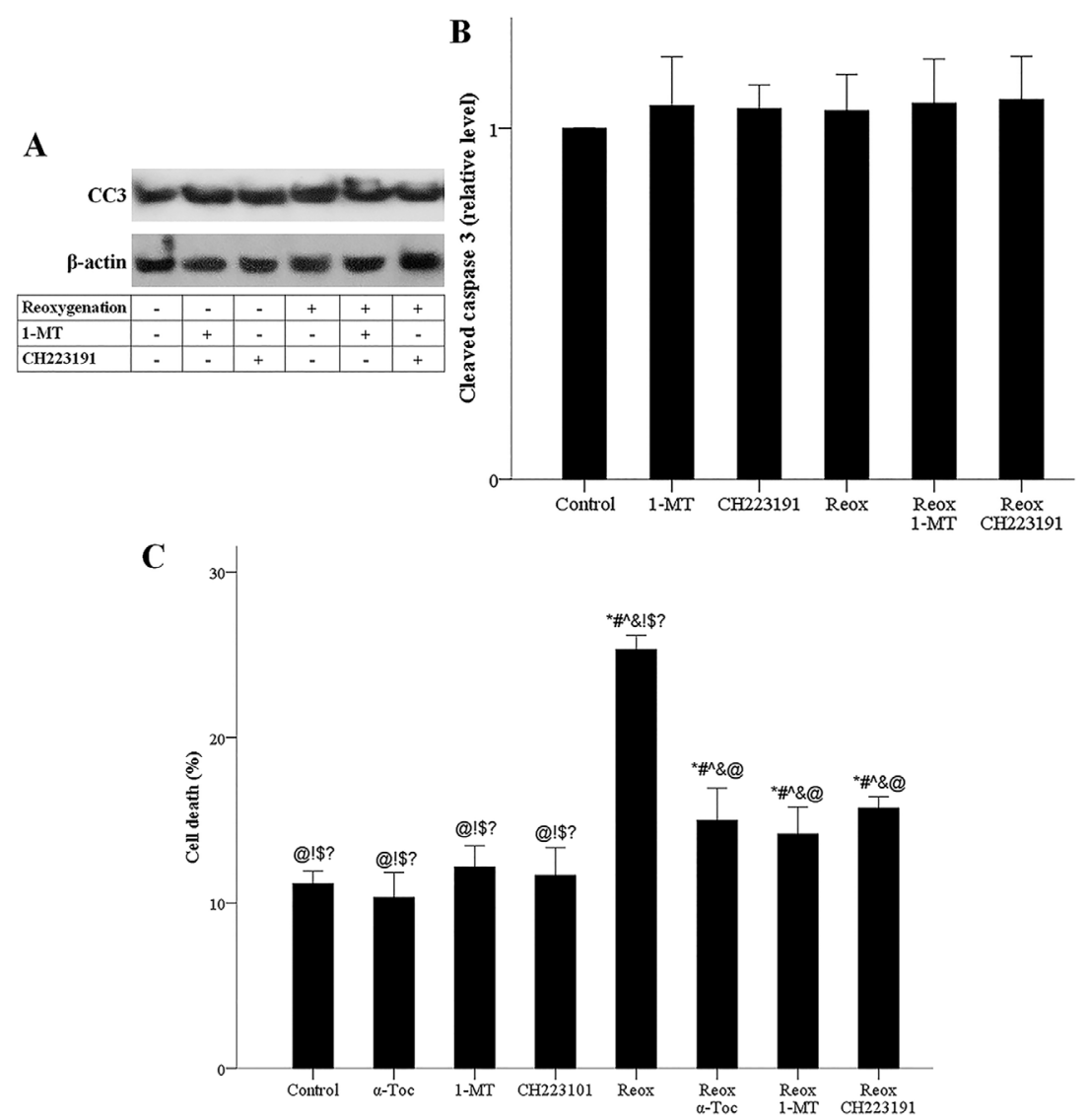

Figure 8. Effect of reox in the presence or absence of the IDO inhibitor 1-MT or the AhR inhibitor CH223191 on CC3 and ferroptotic cell death. (A) CC3 protein expression levels and (B) semi-quantification. (C) $\mathrm{LDH}$ release assay indicating cell death. ${ }^{\mathrm{P}} \mathrm{P}<0.05$ vs. control; ${ }^{\sharp \mathrm{P}}<0.05$ vs. control with $\alpha$-Toc; ${ }^{\wedge} \mathrm{P}<0.05$ vs. control with 1-MT; ${ }^{\circledR} \mathrm{P}<0.05$ vs. control with $\mathrm{CH} 223191 ;{ }^{\circledR} \mathrm{P}<0.05$ vs. Reox; 'P $<0.05$ vs. Reox with $\alpha-\mathrm{Toc} ;{ }^{\$} \mathrm{P}<0.05$ vs. Reox with 1-MT; ${ }^{?} \mathrm{P}<0.05$ vs. Reox with CH223191. 1-MT, 1-DL-methyltryptophan; $\alpha$-Toc, $\alpha$-tocopherol; AhR, aryl-hydrocarbon receptor; CC3, cleaved caspase-3; IDO, indoleamine 2,3-dioxygenase 1; Reox, reoxygenation.
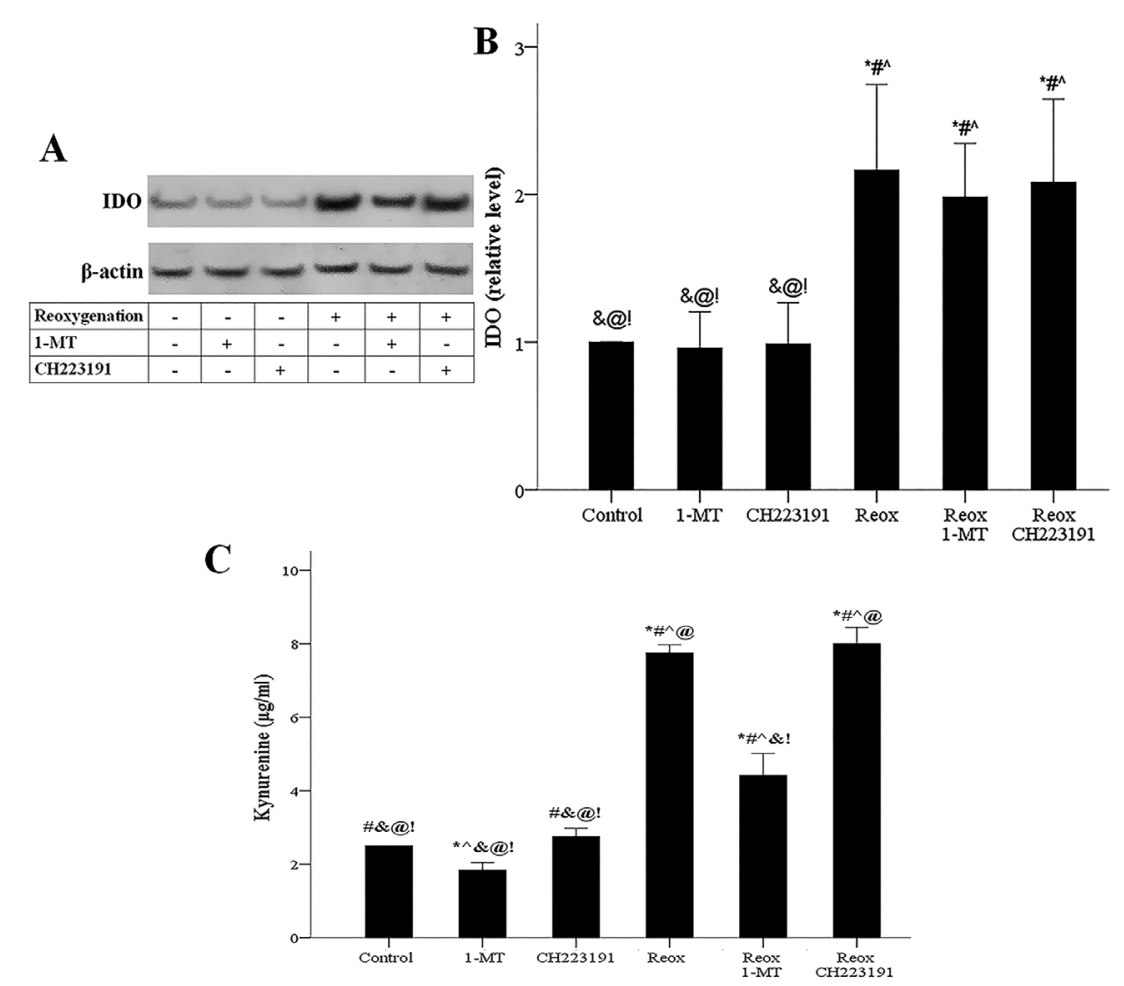

Figure 9. Effect of reox in the presence or absence of the IDO inhibitor 1-MT or the AhR inhibitor CH223191 on IDO expression and kynurenine production. (A) Protein expression of IDO and (B) semi-quantification. (C) Kynurenine production assessed by its concentration in the cell culture supernatant. ${ }^{\text {"P }}<0.05$ vs. control; ${ }^{\sharp} \mathrm{P}<0.05$ vs. control with $1-\mathrm{MT}$; ${ }^{\wedge} \mathrm{P}<0.05$ vs. control with $\mathrm{CH} 223191 ;{ }^{\circledR} \mathrm{P}<0.05$ vs. Reox; ${ }^{\circledR} \mathrm{P}<0.05$ vs. Reox with 1 -MT; ! $<<0.05$ vs. Reox with CH223191. 1-MT, 1-DL-methyltryptophan; AhR, aryl-hydrocarbon receptor; IDO, indoleamine 2,3-dioxygenase 1; Reox, reoxygenation. 

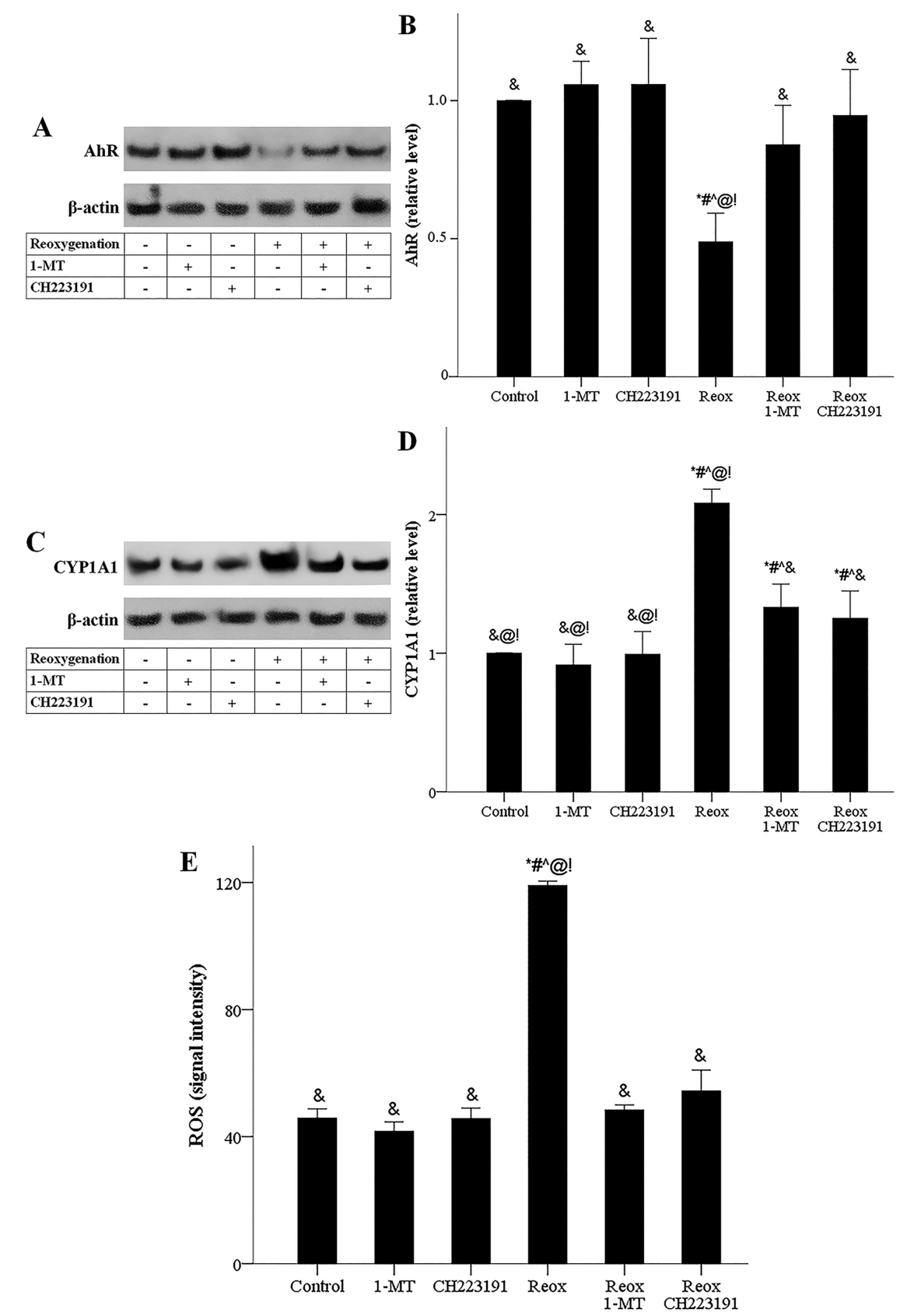

Figure 10. Effect of reox in the presence or absence of the IDO inhibitor 1-MT or the AhR inhibitor CH223191 on AhR level, CYP1A1 expression and ROS generation. (A) AhR protein levels and (B) semi-quantification. (C) CYP1A1 protein levels and (D) semi-quantification. (E) ROS levels. "P< $<0.05$ vs. control; ${ }^{\sharp} \mathrm{P}<0.05$ vs. control with $1-\mathrm{MT}$; ${ }^{\wedge} \mathrm{P}<0.05$ vs. control with $\mathrm{CH} 223191 ;{ }^{\circledR} \mathrm{P}<0.05$ vs. Reox; ${ }^{\circledR} \mathrm{P}<0.05$ vs. Reox with $1-\mathrm{MT} ;$ ! $^{\mathrm{P}}<0.05$ vs. Reox with $\mathrm{CH} 223191$. 1-MT, 1-DL-methyltryptophan; AhR, aryl-hydrocarbon receptor; CYP1A1, cytochrome P450 family 1 subfamily A polypeptide 1; IDO, indoleamine 2,3-dioxygenase 1 ; ROS, reactive oxygen species; Reox, reoxygenation.

mitochondrial membrane permeability, Bax contributes to the intrinsic apoptotic pathway $(19,22)$. DR5, also known as TNF-related apoptosis-inducing ligand receptor 2, sensitizes cells to the extrinsic apoptotic pathway (22). The role of IDO in sensitizing cultured RPTECs subjected to I-R injury to the extrinsic apoptotic pathway has been detected previously (23). Eventually, the present study observed that anoxia causes apoptotic cell death by activating the caspase-3, in which all the apoptotic pathways converge (19). The fact that anoxia-induced IDO upregulation is responsible for the aforementioned molecular events was evaluated and confirmed using the IDO inhibitor 1-MT, which inhibited all the described pathway components.
Another pro-apoptotic factor that plays a significant role in I-R-induced apoptosis is transcription factor p53. Silencing of p53 with siRNA attenuates ischemic acute kidney injury (24). In accordance with a previous study (22), the present study reported that anoxia-induced ATF4-upregulation is accompanied by increased ATF3 expression. ATF3 upregulates $\mathrm{p} 53$ by increasing its gene transcription or by inhibiting p53 proteasomal degradation through direct interaction with p53 or with the mouse double minute 2 homolog (MDM2) (25-27). Indeed, in the current study, anoxia upregulated p53. p53 contributes to the observed increase of Bax and DR5 expression since p53 transcribes several pro-apoptotic genes, including the Bax and DR5 $(28,29)$. 1-MT also inhibited all the aforementioned 


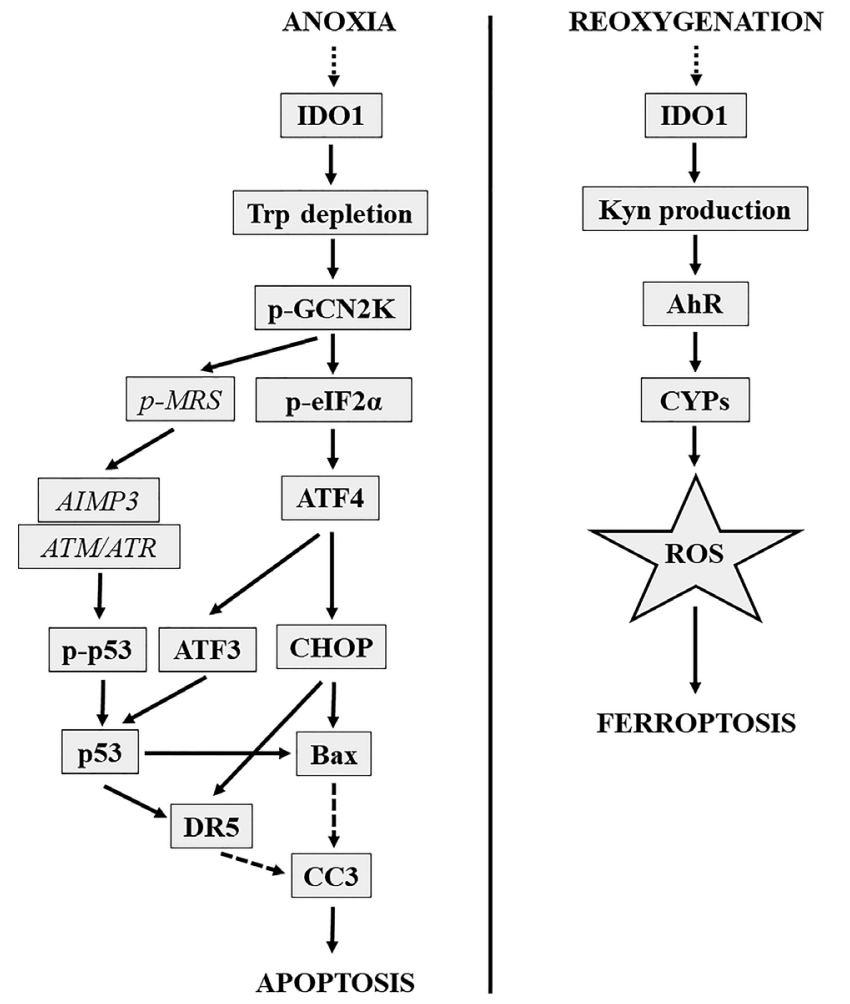

Figure 11. IDO-mediated anoxia-induced apoptosis and reoxygenation-induced ferroptosis molecular pathways. IDO-mediated anoxia-induced apoptotic molecular pathway is depicted on the left. IDO-mediated reoxygenation-induced ferroptotic molecular pathway is depicted on the right AIMP3/p18, aminoacyl-tRNA synthetase-interacting multifunctional protein-3/p18; AhR, aryl-hydrocarbon receptor; ATF3, activating transcription factor 3; ATF4, activating transcription factor 4; ATM/ATR, ataxia-telangiectasia mutated/ataxia-telangiectasia and $\mathrm{Rad} 3$ related protein complex; CHOP, C/EBP homologous protein; CC3, cleaved caspase-3; CYP1A1, cytochrome P450 family 1 subfamily A polypeptide 1; DR5, death receptor 5 IDO, indoleamine 2,3-dioxygenase 1; Kyn, kynurenine; p-, phosphorylated; eIF2a, eukaryotic translation initiation factor- $2 \alpha$; GCN2K, general control nonderepressible-2 kinase; MRS, methionyl-tRNA synthetase; p53, p53; ROS, reactive oxygen species; Trp, tryptophan.

pathway components, confirming that anoxia-induced IDO-overexpression is responsible for p53 upregulation.

Another common mechanism of p53 upregulation involves its phosphorylation at Ser15 by the ataxia-telangiectasia mutated (ATM)/ataxia-telangiectasia and Rad3-related protein (ATR) complex. This phosphorylation leads to 553 dissociation from the MDM2, saving p53 from proteasomal degradation (29). The present study demonstrated that anoxia enhanced p53 phosphorylation, while 1-MT significantly decreased this phosphorylation. A previous study reported that ATM/ATR forms a complex with aminoacyl-tRNA synthetase-interacting multifunctional protein-3/p18 (AIMP3/p18) in the nucleus in order to phosphorylate 533 (30). However, AIMP3/p18 remains in the cytoplasm in a complex with methionyl-tRNA synthetase (MRS). Activated GCN2K phosphorylates MRS, allowing AIMP3/p18 release. In its turn AIMP3/p18 translocates into the nucleus and interacts with ATM/ATR (31). Collectively, the pathways involved in anoxia-induced IDO-mediated apoptosis are depicted in Fig. 11.

The exact mechanism that induces IDO expression under anoxic conditions was not evaluated in the present study. However, in dendritic cells, anoxia induces ATP release into the extracellular space where it is converted to adenosine. Free adenosine induces IDO expression through the adenosine A3 receptor (32). Also, extracellular ATP can upregulate IDO in mesenchymal cells directly through purinergic receptors (33). Since ATP release is a typical response to various types of stress and in numerous cell types (34), the possibility of extracellular ATP-induced IDO expression in RPTECs subjected to I-R deserves evaluation in future studies.

The present study reported that reoxygenation induces ferroptotic and not apoptotic cell death, which is in accordance with previous studies (12-14). ROS-overproduction is a prerequisite for ferroptotic cell death (17). Notably, RPTECs may be particularly vulnerable to ROS since they fail to upregulate certain anti-oxidant defense mechanisms during reoxygenation (35). A source of cellular ROS is the cytochrome P450 superfamily (CYPs) enzymes, which produce ROS during the oxidation of their substrates (36). Experimental models of heart or liver I-R injury show that inhibition of CYPs decreases ROS production and organ dysfunction $(37,38)$. Certain CYPs, particularly CYP1A1, CYP1A2 and CYP1B1, are transcriptional targets of AhR (39). Notably, in experimental models of lung or heart I-R injury, inhibition of AhR was beneficial $(40,41)$. Also, a recent study has shown that in the context of RPTECs, the primary source of ROS for reoxygenation-induced ferroptosis are the CYPs, which are upregulated due to AhR activation (10). AhR is activated by various exogenous and endogenous ligands with kynurenine, a product of tryptophan catabolism by IDO, being one of the endogenous AhR ligands $(7,42)$.

In the present study, reoxygenation upregulated IDO mRNA level and protein expression. As expected, the subsequent kynurenine production activated AhR. It should be noted that when AhR is activated, it becomes vulnerable to proteasomal degradation resulting in lower total cellular levels $(20,21)$. The reoxygenation-induced AhR activation was also confirmed by the increase of the AhR transcriptional target CYP1A1. CYP1A1-upregulation was accompanied by ROS-overproduction, which resulted in ferroptotic cell death. To establish the role of IDO in the aforementioned pathway, the IDO inhibitor 1-MT was added, which suppressed reoxygenation-induced kynurenine production, AhR activation, CYP1A1 expression, ROS generation and eventually cell ferroptosis. The AhR inhibitor CH223191 blocked reoxygenation-induced AhR activation, CYP1A1 expression, ROS generation and ultimately cell ferroptosis. The latter confirms that, in cells subjected to reoxygenation, IDO-upregulation induces cell ferroptosis through the AhR pathway. Collectively, the molecular pathway involved in the reoxygenation-induced IDO-mediated ferroptosis is depicted in Fig. 11.

From a teleological perspective, the upregulation of IDO under anoxia- or reoxygenation-induced stress should be part of an adaptive mechanism aiming to protect the cell from the noxious insult. As with most adaptive mechanisms, depending on the intensity or the duration of the noxious insult, there may be limits in the ability of IDO to protect the cell. There are numerous such paradigms of adaptive responses, such as the genotoxic stress response, the endoplasmic reticulum stress response, the amino acid deprivation stress, autophagy or the ferroptotic mechanism $(6,22,43,44)$. These attempt to restore cellular homeostasis against various stressors. 
However, if the insult is too intense or lasts too long, the same adaptive responses eventually lead to cell death $(6,22,43,44)$. It seems that RPTECs are so vulnerable to anoxia and reoxygenation that at the time points used in the present study that they committed to death. It should be noted that the time points selected for the current experiments were based on the time needed for cell death. It is possible that a shorter exposure of RPTECs to anoxia had been used, then a protective role of IDO would be revealed. It was demonstrated that IDO upregulated p53. Hypothetically, a shorter time of exposure to anoxia may have resulted in p53-induced p21-upregulation, which has an anti-apoptotic effect (45). For instance, albeit in other cell types, a study showed that under hypoxic conditions, activation of GCN2K upregulates p53, resulting in cell cycle arrest through p21-overexpression. However, p21 downregulates Bax, ultimately reducing apoptosis (46). On the contrary, at the time points used in the present study, p53 induced Bax and DR5 expression and eventually apoptosis. Certainly, due to the complexity of the pathways evaluated in the current study, this subject deserves further evaluation. However, in clinical practice, the ischemic insult that may cause acute kidney injury usually lasts longer than the time applied in the present study.

The lack of in vivo verification of the experiments is a limitation of the current study. However, the in vitro approach allowed us to apply strict experimental conditions and evaluate the two pathophysiologically distinct phases of I-R injury, ischemia and reperfusion, separately. Thus, the present study could be considered as a starting point for further in vivo studies.

Besides further clarifying the molecular mechanisms involved in I-R injury, another important finding of the present study is that both ischemia and reperfusion share a common feature; IDO upregulation. This raises the opportunity to intervene in both phases of I-R injury at once with a single therapy. Notably, efforts to interfere with I-R injury by altering tryptophan levels are feasible by administering tryptophan or applying a tryptophan-free diet. Tryptophan is an essential amino acid not synthesized by human cells, and its concentration is the lowest among all the amino acids. In humans, a 2-day low tryptophan intake results in tryptophan depletion (47). However, according to the current results, tryptophan supplementation is expected to alleviate apoptosis during the ischemic phase by decreasing GCN2K activation. During the reperfusion phase, tryptophan supplementation is expected to worsen ferroptosis by increasing kynurenine production and AhR activation. On the other hand, tryptophan depletion is expected to ameliorate ferroptosis during reperfusion and increase apoptosis during ischemia. Thus, inhibition of IDO seems to be a more reliable approach for attenuating I-R injury. Of note, various IDO inhibitors have already been developed and tested in human clinical trials for cancer immunotherapy (48).

In conclusion, in RPTECs, both anoxia and reoxygenation upregulate IDO, which in turn induces GCN2K-mediated apoptosis and AhR-mediated ferroptosis, respectively. The inhibition of IDO may prove a useful therapeutic strategy for preventing or attenuating I-R injury.

\section{Acknowledgements}

Not applicable.

\section{Funding}

No funding was received.

\section{Availability of data and materials}

The datasets used and/or analyzed during the current study are available from the corresponding author on reasonable request.

\section{Authors' contributions}

TE designed the study. GP and TE performed the experiments, and collected the data. TE and GP confirm the authenticity of all raw data. TE interpreted the data with help from GP, SG, VL and IS. TE, GP, SG, VL and IS analyzed the results. TE wrote the manuscript with help from GP. All authors read and approved the final manuscript.

\section{Ethics approval and consent to participate}

Not applicable.

\section{Patient consent for publication}

Not applicable.

\section{Competing interests}

The authors declare that they have no competing interests.

\section{References}

1. Wu MY, Yiang GT, Liao WT, Tsai AP, Cheng YL, Cheng PW, $\mathrm{Li} \mathrm{CY}$ and Li CJ: Current mechanistic concepts in ischemia and reperfusion injury. Cell Physiol Biochem 46: 1650-1667, 2018.

2. Bonventre JV and Yang L: Cellular pathophysiology of ischemic acute kidney injury. J Clin Invest 121: 4210-4221, 2011.

3. King NJ and Thomas SR: Molecules in focus: Indoleamine 2,3-dioxygenase. Int J Biochem Cell Biol 39: 2167-2172, 2007.

4. Munn DH, Zhou M, Attwood JT, Bondarev I, Conway SJ, Marshall B, Brown C and Mellor AL: Prevention of allogeneic fetal rejection by tryptophan catabolism. Science 281: 1191-1193, 1998.

5. Eleftheriadis T, Pissas G, Antoniadi G, Spanoulis A, Liakopoulos V and Stefanidis I: Indoleamine 2,3-dioxygenase increases p53 levels in alloreactive human $\mathrm{T}$ cells, and both indoleamine 2,3-dioxygenase and p53 suppress glucose uptake, glycolysis and proliferation. Int Immunol 26: 673-684, 2014.

6. Castilho BA, Shanmugam R, Silva RC, Ramesh R, Himme BM and Sattlegger E: Keeping the eIF2 alpha kinase Gcn2 in check. Biochim Biophys Acta 1843: 1948-1968, 2014.

7. Mezrich JD, Fechner JH, Zhang X, Johnson BP, Burlingham WJ and Bradfield CA: An interaction between kynurenine and the aryl hydrocarbon receptor can generate regulatory $\mathrm{T}$ cells. J Immunol 185: 3190-3198, 2010.

8. Eleftheriadis T, Pissas G, Liakopoulos V and Stefanidis I: IDO decreases glycolysis and glutaminolysis by activating GCN2K, while it increases fatty acid oxidation by activating AhR, thus preserving $\mathrm{CD}^{+} \mathrm{T}$ cell survival and proliferation. Int $\mathrm{J}$ Mol Med 42: 557-568, 2018.

9. Mohib K, Wang S, Guan Q, Mellor AL, Sun H, Du C and Jevnikar AM: Indoleamine 2,3-dioxygenase expression promotes renal ischemia-reperfusion injury. Am J Physiol Renal Physiol 295: F226-F234, 2008.

10. Eleftheriadis T, Pissas G, Filippidis G, Liakopoulos V and Stefanidis I: Reoxygenation induces reactive oxygen species production and ferroptosis in renal tubular epithelial cells by activating aryl hydrocarbon receptor. Mol Med Rep: Nov 10, 2020 (Epub ahead of print). doi: 10.3892/mmr.2020.11679. 
11. Khan S, Cleveland RP, Koch CJ and Schelling JR: Hypoxia induces renal tubular epithelial cell apoptosis in chronic renal disease. Lab Invest 79: 1089-1099, 1999.

12. Eleftheriadis T, Pissas G, Antoniadi G, Liakopoulos V and Stefanidis I: cell death patterns due to warm ischemia or reperfusion in renal tubular epithelial cells originating from human, mouse, or the native hibernator hamster. Biology (Basel) 7: 7, 2018

13. Eleftheriadis T, Pissas G, Liakopoulos V and Stefanidis I: Factors that may protect the native hibernator syrian hamster renal tubular epithelial cells from ferroptosis due to warm anoxia-reoxygenation. Biology (Basel) 8: 8, 2019.

14. Linkermann A, Skouta R, Himmerkus N, Mulay SR, Dewitz C, De Zen F, Prokai A, Zuchtriegel G, Krombach F, Welz PS, et al: Synchronized renal tubular cell death involves ferroptosis. Proc Natl Acad Sci USA 111: 16836-16841, 2014.

15. Jia L, Schweikart K, Tomaszewski J, Page JG, Noker PE, Buhrow SA, Reid JM, Ames MM and Munn DH: Toxicology and pharmacokinetics of 1-methyl-d-tryptophan: Absence of toxicity due to saturating absorption. Food Chem Toxicol 46: 203-211, 2008

16. Kim SH, Henry EC, Kim DK, Kim YH, Shin KJ, Han MS Lee TG, Kang JK, Gasiewicz TA, Ryu SH, et al: Novel compound 2-methyl-2H-pyrazole-3-carboxylic acid (2-methyl-4-o-tolylaz o-phenyl)-amide (CH-223191) prevents 2,3,7,8-TCDD-induced toxicity by antagonizing the aryl hydrocarbon receptor. Mol Pharmacol 69: 1871-1878, 2006.

17. Stockwell BR, Friedmann Angeli JP, Bayir H, Bush AI, Conrad M, Dixon SJ, Fulda S, Gascón S, Hatzios SK, Kagan VE, et al: Ferroptosis: A regulated cell death nexus linking metabolism, redox biology, and disease. Cell 171: 273-285, 2017.

18. Lobner D: Comparison of the LDH and MTT assays for quantifying cell death: Validity for neuronal apoptosis? J Neurosci Methods 96: 147-152, 2000.

19. Fadeel B and Orrenius S: Apoptosis: A basic biological phenomenon with wide-ranging implications in human disease. J Intern Med 258: 479-517, 2005

20. Pollenz RS: The mechanism of AH receptor protein down-regulation (degradation) and its impact on $\mathrm{AH}$ receptor-mediated gene regulation. Chem Biol Interact 141: 41-61, 2002.

21. Yang SC, Wu CH, Tu YK, Huang SY and Chou PC: Exposure to 2,3,7,8-tetrachlorodibenzo-p-dioxin increases the activation of aryl hydrocarbon receptor and is associated with the aggressiveness of osteosarcoma MG-63 osteoblast-like cells. Oncol Lett 16: 3849-3857, 2018.

22. $\mathrm{Hu} \mathrm{H}$, Tian M, Ding $\mathrm{C}$ and $\mathrm{Yu}$ S: The C/EBP homologous protein (CHOP) transcription factor functions in endoplasmic reticulum stress-induced apoptosis and microbial infection. Front Immunol 9: 3083, 2019.

23. Mohib K, Guan Q, Diao H, Du C and Jevnikar AM: Proapoptotic activity of indoleamine 2,3-dioxygenase expressed in renal tubular epithelial cells. Am J Physiol Renal Physiol 293: F801-F812, 2007.

24. Molitoris BA, Dagher PC, Sandoval RM, Campos SB Ashush H, Fridman E, Brafman A, Faerman A, Atkinson SJ, Thompson JD, et al: siRNA targeted to p53 attenuates ischemic and cisplatin-induced acute kidney injury. J Am Soc Nephrol 20: 1754-1764, 2009.

25. You Z, Xu J, Li B, Ye H, Chen L, Liu Y and Xiong X: The mechanism of ATF3 repression of epithelial-mesenchymal transition and suppression of cell viability in cholangiocarcinoma via p53 signal pathway. J Cell Mol Med 23: 2184-2193, 2019.

26. Wang Z, He Y, Deng W, Lang L, Yang H, Jin B, Kolhe R, Ding HF, Zhang J, Hai T, et al: Atf3 deficiency promotes genome instability and spontaneous tumorigenesis in mice. Oncogene 37 18-27, 2018.

27. Li X, Guo M, Cai L, Du T, Liu Y, Ding HF, Wang H, Zhang J, Chen $\mathrm{X}$ and Yan C: Competitive ubiquitination activates the tumor suppressor p53. Cell Death Differ 27: 1807-1818, 2020.

28. Taketani K, Kawauchi J, Tanaka-Okamoto M, Ishizaki H, Tanaka Y, Sakai T, Miyoshi J, Maehara Y and Kitajima S: Key role of ATF3 in p53-dependent DR5 induction upon DNA damage of human colon cancer cells. Oncogene 31: 2210-2221, 2012

29. Aubrey BJ, Kelly GL, Janic A, Herold MJ and Strasser A: How does $\mathrm{p} 53$ induce apoptosis and how does this relate to $\mathrm{p} 53$-mediated tumour suppression? Cell Death Differ 25: 104-113, 2018.
30. Park BJ, Kang JW, Lee SW, Choi SJ, Shin YK, Ahn YH, Choi YH, Choi D, Lee KS and Kim S: The haploinsufficient tumor suppressor p18 upregulates p53 via interactions with ATM/ATR. Cell 120: 209-221, 2005

31. Kwon NH, Kang T, Lee JY, Kim HH, Kim HR, Hong J, Oh YS, Han JM, Ku MJ, Lee SY, et al: Dual role of methionyl-tRNA synthetase in the regulation of translation and tumor suppressor activity of aminoacyl-tRNA synthetase-interacting multifunctional protein-3. Proc Natl Acad Sci USA 108: 19635-19640, 2011.

32. Song X, Zhang Y, Zhang L, Song W and Shi L: Hypoxia enhances indoleamine 2,3-dioxygenase production in dendritic cells. Oncotarget 9: 11572-11580, 2018.

33. Lotfi R, Steppe L, Hang R, Rojewski M, Massold M, Jahrsdörfer B and Schrezenmeier H: ATP promotes immunosuppressive capacities of mesenchymal stromal cells by enhancing the expression of indoleamine dioxygenase. Immun Inflamm Dis 6: 448-455, 2018.

34. Dosch M, Gerber J, Jebbawi F and Beldi G: Mechanisms of ATP release by inflammatory cells. Int J Mol Sci 19: 19, 2018.

35. Eleftheriadis T,Pissas G, Nikolaou E, Filippidis G, Liakopoulos V and Stefanidis I: Mistimed H2S upregulation, Nrf2 activation and antioxidant proteins levels in renal tubular epithelial cells subjected to anoxia and reoxygenation. Biomed Rep 13: 3, 2020.

36. Hrycay EG and Bandiera SM: Monooxygenase, peroxidase and peroxygenase properties and reaction mechanisms of cytochrome P450 Enzymes. Adv Exp Med Biol 851: 1-61, 2015.

37. Ishihara Y, Sekine M, Nakazawa M and Shimamoto N: Suppression of myocardial ischemia-reperfusion injury by inhibitors of cytochrome P450 in rats. Eur J Pharmacol 611: 64-71, 2009.

38. Shaik IH and Mehvar R: Effects of cytochrome p450 inhibition by cimetidine on the warm hepatic ischemia-reperfusion injury in rats. J Surg Res 159: 680-688, 2010.

39. Nebert DW, Dalton TP, Okey AB and Gonzalez FJ: Role of aryl hydrocarbon receptor-mediated induction of the CYP1 enzymes in environmental toxicity and cancer. J Biol Chem 279: 23847-23850, 2004

40. Cuartero MI,Ballesteros I, de la Parra J, Harkin AL, Abautret-Daly A, Sherwin E, Fernández-Salguero P, Corbí AL, Lizasoain I and Moro MA: L-kynurenine/aryl hydrocarbon receptor pathway mediates brain damage after experimental stroke. Circulation 130: 2040-2051, 2014

41. Couroucli XI, Liang YW, Jiang W, Barrios R and Moorthy B: Attenuation of oxygen-induced abnormal lung maturation in rats by retinoic acid: Possible role of cytochrome P4501A enzymes. J Pharmacol Exp Ther 317: 946-954, 2006.

42. Stejskalova L, Dvorak Z and Pavek P: Endogenous and exogenous ligands of aryl hydrocarbon receptor: Current state of art. Curr Drug Metab 12: 198-212, 2011.

43. Bartek J and Lukas J: DNA damage checkpoints: From initiation to recovery or adaptation. Curr Opin Cell Biol 19: 238-245, 2007.

44. Galluzzi L, Bravo-San Pedro JM, Vitale I, Aaronson SA, Abrams JM, Adam D, Alnemri ES, Altucci L, Andrews D, Anni cchiarico-Petruzzelli M, et al: Essential versus accessory aspects of cell death: Recommendations of the NCCD 2015. Cell Death Differ 22: 58-73, 2015.

45. Mirzayans R, Andrais B, Kumar P and Murray D: Significance of wild-type p53 signaling in suppressing apoptosis in response to chemical genotoxic agents: Impact on chemotherapy outcome. Int J Mol Sci 18: 928, 2017.

46. Liu Y, László C, Liu Y, Liu W, Chen X, Evans SC and Wu S Regulation of $\mathrm{G}(1)$ arrest and apoptosis in hypoxia by PERK and GCN2-mediated eIF2 $\alpha$ phosphorylation. Neoplasia 12: 61-68, 2010.

47. Ellenbogen MA, Young SN, Dean P, Palmour RM and Benkelfat C: Mood response to acute tryptophan depletion in healthy volunteers: Sex differences and temporal stability. Neuropsychopharmacology 15: 465-474, 1996.

48. Le Naour J, Galluzzi L, Zitvogel L, Kroemer G and Vacchelli E: Trial watch: IDO inhibitors in cancer therapy. OncoImmunology 9: $1777625,2020$.

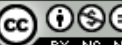

This work is licensed under a Creative Commons Attribution-NonCommercial-NoDerivatives 4.0 International (CC BY-NC-ND 4.0) License. 